\title{
Índice de reinserción laboral tras un programa de rehabilitación cardiaca
}

\section{Index reinsertion working after cardiac rehabilitation program}

\author{
Inés Pérez Leal', Rodrigo Tallón Moreno², Miguel González Valdayo³ \\ 1. Médico-Inspector de la Dirección Provincial del INSS de Sevilla. España \\ 2. Médico del Servicio de Rehabilitación del Hospital Virgen Macarena de Sevilla. España \\ 3. Cardiólogo del Servicio de Rehabilitación del Hospital Virgen Macarena de Sevilla. España \\ Recibido: 05-06-14 \\ Aceptado: 17-06-14

\section{Correspondencia} \\ Inés Pérez Leal \\ Correo electrónico: ines.perez2@seg-social.es, inesperezleal5@gmail.com
}

Resumen

El Infarto Agudo de Miocardio (IAM) es la Entidad que produce mayor morbimortalidad en nuestra Sociedad. Afecta a la Calidad de vida del Paciente y a su Situación Laboral, siendo responsable de un alto porcentaje de Incapacidades Permanentes. Así como también se encuentran entre las principales Causas de Incapacidad Temporal.

Los datos de Reincorporación laboral tras un IAM varían mucho de unos Países a otros. Así como de unas Comunidades Autónomas a otras en nuestro país.

Se han experimentado grandes Avances Tecnológicos en el campo del Intervencionismo Coronario Percutáneo (ACTP) y la colocación de Endoprótesis Vasculares (STENT). Así como los Programas de Rehabilitación Cardiaca y Prevención Secundaria, con actuaciones en el campo del control de los factores de riesgo cardiovascular, Programas de Entrenamiento físico, intervención psicológica, programas educativos y de valoración sociolaboral han facilitado la Recuperación funcional de los pacientes que han sufrido un IAM con mejoras en la funcionalidad cardiaca y en la capacidad funcional de los pacientes con mejor percepción y calidad de vida, que les permite la reincorporación a sus actividades anteriores a sufrir el evento coronario agudo, entre ellas la laboral.

En un Programa de Actuaciones y de Colaboración conjunta entre la Unidad Médica de la Dirección Provincial de Sevilla del INSS y la Unidad de Rehabilitación Cardiaca y Prevención Secundaria del Hospital Universitario Virgen Macarena de Sevilla, nos planteamos hacer un seguimiento de los pacientes que realizaron estos Programas de Rehabilitación Cardiaca en el año 2011 con vistas a la posibilidad de su Reincorporación laboral.

En nuestro estudio hemos demostrado que los Pacientes que siguen un Programa Multidisciplinar de Rehabilitación Cardiaca, consiguen mejoría en los variables que hemos analizado, con mejoría de su Funcionalidad Cardiaca $(84,3 \%)$ y sensación de Bienestar Físico y Calidad de vida percibida (74\%). Siendo subsidiarios de indicación de Reincorporación Laboral el 63\% de los pacientes incluidos en nuestro Estudio.

Med Segur Trab (Internet) 2014; 60 (235) 370-378

Palabras claves: IAM (Infarto agudo de miocardio), Reincorporación Laboral, Rebabilitación Cardiaca. 


\section{Abstract}

The Acute Myocardial Infarctio (AMI) is the entity that produces the greatest morbidity in our society. It affects the quality of life of the patient and their Employment Status, responsible for a high percentage of Permanent Disabilities. And also among the major causes of temporary disability.

Data Return to work after an AMI, vary widely from country to country. And from one Autonomous Community to another in our country.

They have experienced great technological advances in the field of percutaneous coronary intervention (PTCA) and Stent placement vascular (STENT). And Cardiac control of cardiovascular risk factors, physical training programs, psychological intervention, educational and socio-assessment have facilitated the functional recovery of patients with improvements in cardiac and functional capacity of patients with better insight and quality of life, allowing them to return to their previous suffering acute coronary event, including labor activities functionality.

In a program of performances and joint collaboration between the Medical Unit of the Provincial Directorate of Sevilla INSS and Unit Cardiac Rehabilitation and Secondary Prevention of Hospital Virgen Macarena in Seville, we propose to monitor the patients undergoing these programs Cardiac Rehabilitation in 2011 with a view to the possibility of their return to work.

In our study we have shown that patients who follow a Multidisciplinary Cardiac Rehabilitation Program, get improvement in the variables listed above, with improvement in their functionality cardiac (84,3\%) and feeling of Physical fitness and perceived quality of life (74\%). Being labor subsidiary indication reinstatement $63 \%$ of patients included in our study.

Med Segur Trab (Internet) 2014; 60 (235) 370-378

Keywords: AMI (Acute Myocardial Infarction), Reinstatement Labor, Cardiac Rehabilitation. 


\section{INTRODUCCIÓN}

El Infarto Agudo de Miocardio (IAM) es la Entidad que produce mayor morbimortalidad en nuestra Sociedad. Afecta a la Calidad de vida del Paciente y a su Situación Laboral, siendo responsable de un alto porcentaje de Incapacidades Permanentes.

Las Enfermedades Cardiovasculares se encuentran entre las principales Causas de Baja Laboral en nuestro País, tras la Lumbalgia, la Depresión y los Trastornos Musculoesqueléticos. En España se registra cada año 23.000 Bajas Laborales por Enfermedad Cardiovascular.

Los datos de Reincorporación laboral tras un IAM, varían mucho de unos Países a otros. Asi como de unas Comunidades Autónomas a otras en nuestro país.

Hemos de considerar que se han experimentado grandes Avances Tecnológicos en el campo del Intervencionismo Coronario Percutáneo (ACTP) y la colocación de Endoprótesis Vasculares (STENT). Así como que se han introducido los PRC( Programas de Rehabilitación Cardiaca y Prevención Secundaria), donde interviene un Equipo Multidisciplinar de Profesionales: Cardiólogo, Rehabilitador, Enfermería, Fisioterapeuta, Terapeutas Ocupacionales, Psicólogos, Dietistas y Trabajadores Sociales.

Realizándose una Intervención que comprende tres Fases.

FASE I: Comprende el manejo del paciente mientras está ingresado en el Hospital.

FASE II: Se realiza tras el Alta Hospitalaria. La duración suele ser de 2-3 meses. Es necesario realizar una ESTRATIFICACIÓN DE RIESGO de los Pacientes, mediante la Valoración Cardiológica (realizando una Ergometría, Ecocardiografia y Valoración de los FRCV). Estableciéndose tres categorías:

\section{BAJO RIESGO:}

Curso Hospitalario sin complicaciones. Ausencia de signos de Isquemia miocárdica. Capacidad Funcional > de 7 METS. Fracción de Eyección mayor del 50\%. Ausencia de Arritmias ventriculares severas.

\section{RIESGO MEDIO:}

Defectos reversibles con el Talio de Esfuerzo. Aparición de Angina. Capacidad Funcional de 5-7 METS. Fracción de Eyección de 35-49\%.

RIESGO ALTO:

Reinfarto. Insuficiencia cardíaca, Depresión del ST > de $2 \mathrm{~mm}$. Fracción de eyección menor del 35\%. Capacidad Funcional menor de 5 METS. Respuesta Hipotensiva al Esfuerzo. Arritmias Ventriculares Malignas.

En esta Fase II también se realizará: Valoración Psicológica (de Depresión, Ansiedad, Pautas de Conducta, Acontecimientos vitales Estresantes) y Valoración Socio-Laboral.

Estos Programas incluyen el Entrenamiento Físico, el Control de los Factores de Riesgo Cardiovasculares, La Intervención Psicológica, Programa Educativo y Valoración Socio-Laboral.

Consiguiéndose a través de los Cambios de Hábitos y Estilo de vida, mejorar la Funcionalidad Cardiaca, la Funcionalidad Física, Reducir la Sintomatología, y mejorar la Calidad de Vida y el buen Estado en general. Facilitando la Recuperación Funcional y permitiendo al paciente Reincorporarse a su Actividad. 


\section{OBJETIVOS}

\section{General}

Cuantificar el número de Pacientes que presentan indicación de Reincorporación Laboral tras el Programa de Rehabilitación Cardiaca.

\section{Específico}

- Relacionar la Reincorporación Laboral según el nivel de Riesgo Cardiovascular

- Identificar la Situación Laboral previa y el número de Pacientes que se Reincorporan.

- Relacionar el Riesgo Inicial y Final de los Pacientes tras la realización del Programa de Rehabilitación Cardiaca con el número de Pacientes que se Reincorporan.

- Describir el número de Pacientes que se les indica Reincorporación Laboral.

- Identificar la Percepción de Calidad de Vida.

- Evaluar la Capacidad Funcional pre y post realización del Programa mediante Prueba de Esfuerzo y Ventriculografía como indicador de modificación de Riesgo Cardiovascular

\section{MATERIAL Y MÉTODO}

\section{Diseño}

Estudio Observacional Descriptivo.

\section{Sujetos y selección de participantes}

La Población Elegible eran los Pacientes incluidos en el Programa de Rehabilitación Cardiaca del HUVM (Hospital Universitario Virgen Macarena de Sevilla) desde 1 de enero de 2011 a 30 julio de 2011.

Se realizó un Muestreo Intencional, incluyendo los Pacientes que tenían los siguientes Criterios para su Inclusión: encontrarse en Edad laboral Activa (hasta 65 años), que hubieran completado el Programa de Rehabilitación Cardiaca (2 sesiones/semana 2 meses).

Y como criterio de Exclusión: los Pacientes del grupo de Bajo Riesgo a los que solo se les indicó la realización del Programa Educacional (1 semana) e Indicación de Reincorporación Laboral. El tamaño muestral fue de 108 Pacientes.

\section{Variables de estudio}

Se ha realizado un Registro con las siguientes Variables: - Edad, Sexo, nivel de Estudios, Estado Civil, tipo de Trabajo (Cualificado/no Cualificado), Situación Laboral.

- Valoración de la FE pretest mediante Ecocardiografía Doppler inicial y Valoración de la FE mediante Ventriculografía final,

- Valoración de la Capacidad Funcional pretest y postest mediante Ergometrías Inicial y Final.

- Grupo de Riesgo Inicial y grupo de Riesgo Final, Reinserción Laboral y Calidad de Vida

(mediante el Cuestionario Español de Calidad de Vida en Pacientes postIAM de Velasco del Barrio). 


\section{Recogida de información}

La Recogida de los Datos se realizo a través de la Historia Clínica de los Pacientes, donde se archivaban datos Socio- demográfico, Pruebas Complementarias, Evaluaciones e Informes que se les ha realizado durante la realización del Programa de Rehabilitación Cardiaca.

\section{Análisis de los datos}

Se realizó un Análisis Descriptivo de todas las Variables en Estudio.

Para Comparar los Resultados de las Ergometrías y Ventriculografías Iníciales y Finales, se realizo la Prueba T de Student y Test de ANOVA para relacionar la Reinserción Laboral con el grupo de Riesgo. Utilizando el programa SSPS 17.0.

\section{Resultados}

El número total de pacientes estudiado que han cumplido con los Criterios de Inclusión han sido 108, con una Edad media de 52,70 años (DT 7,64) siendo el 86,1\% varones, el nivel de Estudios en el 39,8\% eran Estudios Primarios, el 33,3\% Secundarios y un $25 \%$ Universitarios.

El 76,9\% eran casados con un Trabajo no Cualificado en el 57,4\%.

La situación Laboral de los Pacientes en un 54,6\% se encontraba en Incapacidad Temporal (IT).

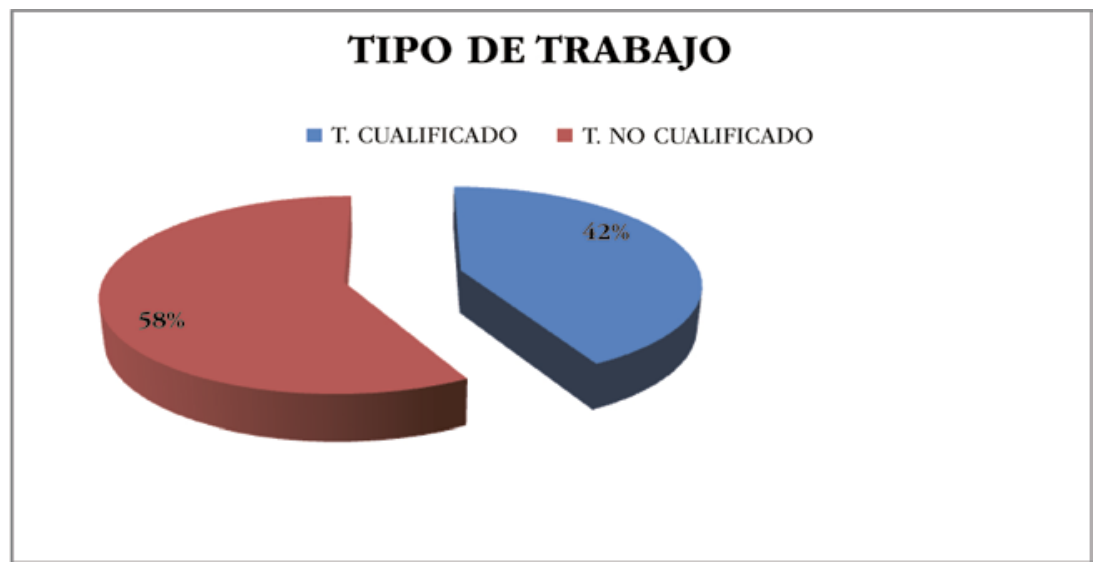




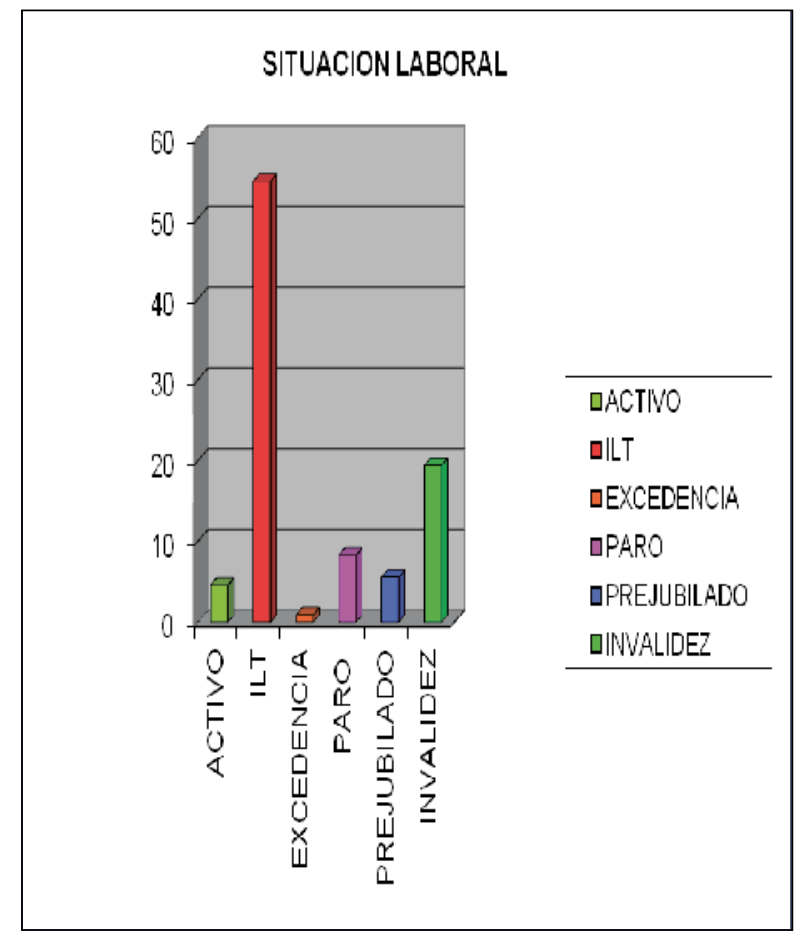

- El Riesgo Inicial de los Pacientes al inicio del Programa era: Bajo el 42,6\%. Moderado el 44,45\% y Alto el 13\% y

- El Riesgo al finalizar el Programa se observa que el número de Pacientes de: Bajo Riesgo es el 84,3\%, Moderado el 9,3\% y Alto un 5,6\%.

El porcentaje de Reincorporación Laboral fue del 63\%.

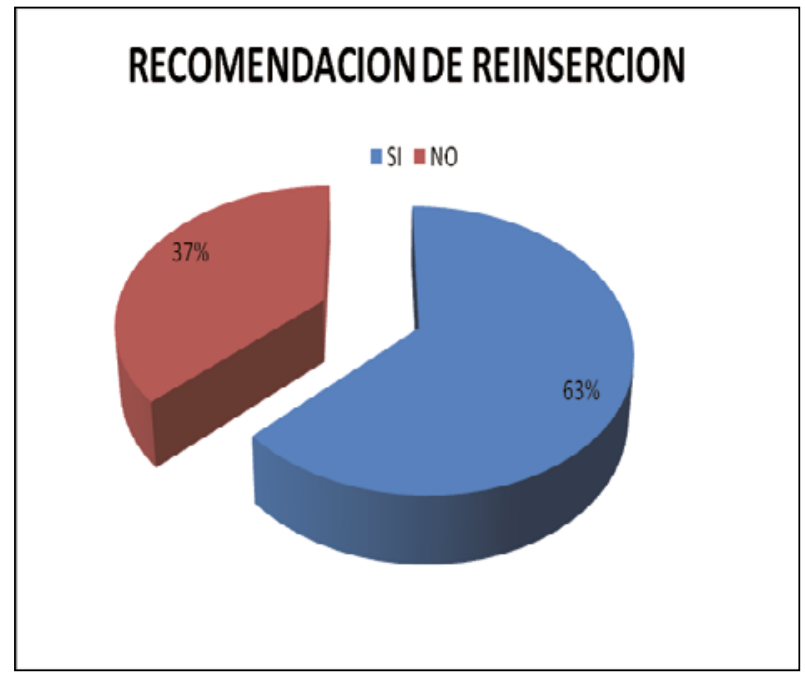




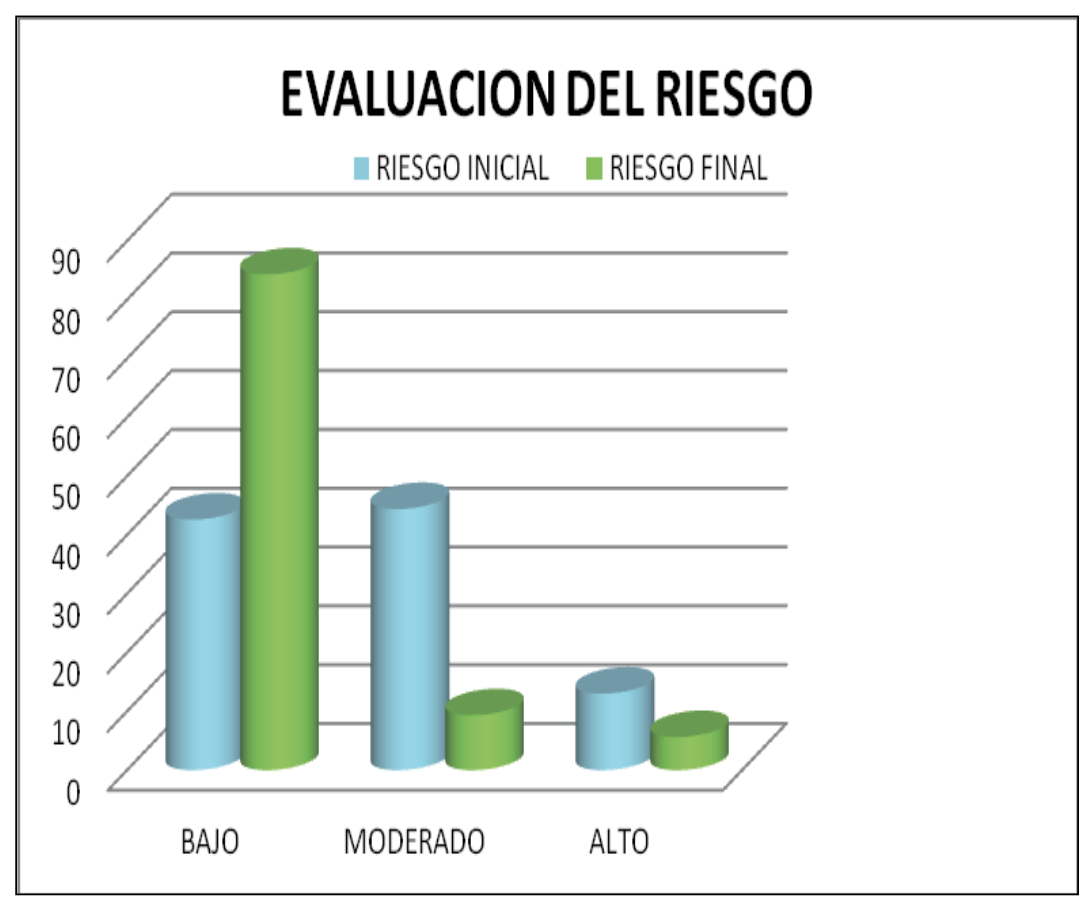

En el Análisis de la Prueba de la T de Student se observó una clara evolución de la Capacidad Funcional de los Pacientes al finalizar el Programa de Rehabilitación Cardiaca, partiendo de unos valores de Ergometría Inicial con un valor medio de 7,55 METS y una Ergometría Final de 9,64 METS (t=-11,5; p=0.000).

Estadísticos de muestras relacionadas

\begin{tabular}{ccccc}
\hline & Media & $\mathbf{N}$ & $\begin{array}{c}\text { Desviación } \\
\text { típ. }\end{array}$ & $\begin{array}{c}\text { Error típ. } \\
\text { de la media }\end{array}$ \\
\hline Par 1 METS.INICIAL & 7,5500 & 108 & 2,35608 &, 22671 \\
METS.FINAL & 9,6435 & 108 & 2,76805 &, 26636 \\
\hline
\end{tabular}

Correlaciones de muestras relacionadas

\begin{tabular}{rccc}
\hline & $\mathbf{N}$ & Correlación & Sig. \\
\hline $\begin{array}{r}\text { Par 1 METS.INICIAL } \\
\text { y METS.FINAL }\end{array}$ & 108 &, 740 &, 000 \\
\hline
\end{tabular}

Así como una gran mejoría de la FEVI (Fracción de Eyección Ventricular izquierda)

Al finalizar el Programa de Rehabilitación Cardiaca, partiendo de una Ventriculografía Inicial con un valor medio de 49,53\% y una Final de 57,20\% $(t=-7,965 ; \mathrm{p}=0.000)$

Estadísticos de muestras relacionadas

\begin{tabular}{|c|c|c|c|c|}
\hline & Media & $\mathbf{N}$ & $\begin{array}{c}\text { Desviación } \\
\text { típ. }\end{array}$ & $\begin{array}{l}\text { Error típ. } \\
\text { de la media }\end{array}$ \\
\hline Par 1 FE.INICIAL & 49,5398 & 108 & 9,98800 & ,96110 \\
\hline FE.FINAL & 57,2056 & 108 & 11,21944 & 1,07959 \\
\hline \multicolumn{5}{|c|}{ Correlaciones de muestras relacionadas } \\
\hline & & $\mathbf{N}$ & Correlación & Sig. \\
\hline Par 1 FE.INICIAL & E.FINAL & 108 &, 560 & ,000 \\
\hline
\end{tabular}


Prueba de muestras relacionadas

\begin{tabular}{|c|c|c|c|c|c|c|c|c|}
\hline & \multicolumn{5}{|c|}{ Diferencias relacionadas } & \multirow{3}{*}{$\mathbf{t}$} & \multirow{3}{*}{ g1 } & \multirow{3}{*}{ Sig. (bilateral) } \\
\hline & \multirow[t]{2}{*}{ Media } & \multirow{2}{*}{$\begin{array}{l}\text { Desviación } \\
\text { tip. }\end{array}$} & \multirow{2}{*}{$\begin{array}{l}\text { Error tip. de } \\
\text { la media }\end{array}$} & \multicolumn{2}{|c|}{$\begin{array}{l}05 \% \text { Intervalo de con- } \\
\text { fianza para la diferencia }\end{array}$} & & & \\
\hline & & & & Inferior & Superior & & & \\
\hline $\begin{array}{l}\text { Par } 1 \text { FE.INICIAL Y } \\
\text { FE. FINAL }\end{array}$ & $-7,66574$ & 10,00218 & ,96246 & $-9,57371$ & $-5,757758$ & $-7,965$ & 107 &, 000 \\
\hline
\end{tabular}

A través del Test de ANOVA:

- Se pudo establecer que la variable Reinserción Laboral estaba relacionada con la pertenencia al grupo de Riesgo Final tras la realización del Programan de Rehabilitación Cardiaca. Y se observó una gran mejoría en la Calidad de Vida.

ANOVA

Reinserción

\begin{tabular}{lrrrcr}
\hline & $\begin{array}{c}\text { Sum of } \\
\text { Squares }\end{array}$ & df & $\begin{array}{c}\text { Mean } \\
\text { Square }\end{array}$ & F & Sig. \\
\hline Between Groups & 7,598 & 2 & 3,799 & 22,989 &, 000 \\
Within Groups & 17,187 & 104 &, 165 & & \\
Total & 24,785 & 106 & & & \\
\hline
\end{tabular}

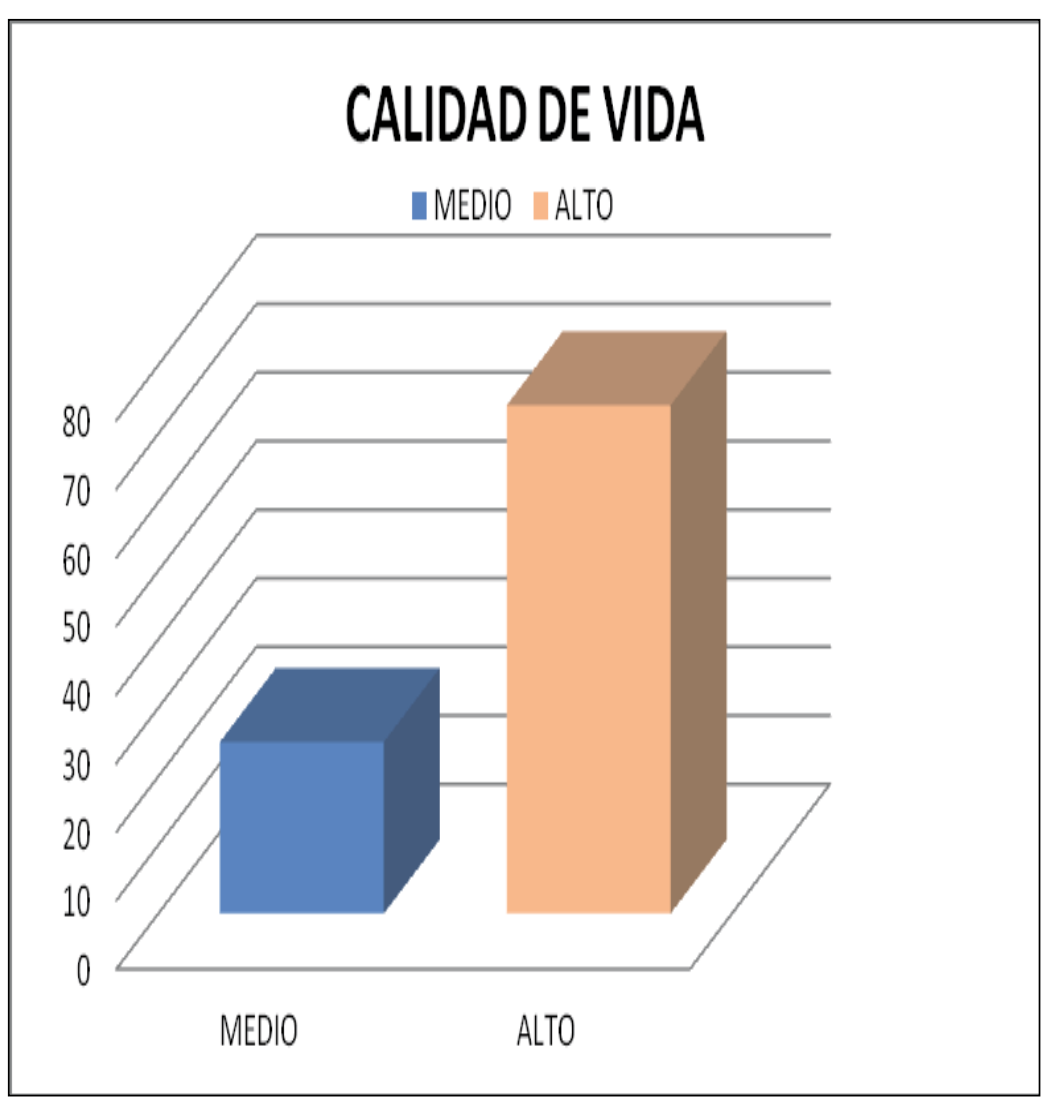




\section{CONCLUSIONES}

En nuestro estudio hemos demostrado que los Pacientes que siguen un Programa Multidisciplinar de Rehabilitación Cardiaca, consiguen mejoría en los variables que hemos analizado, con mejoría de su Funcionalidad Cardiaca (84,3\%) y sensación de Bienestar Físico y Calidad de vida percibida (74\%).

Siendo subsidiarios de indicación de Reincorporación Laboral el 63\% de los pacientes incluidos en nuestro Estudio.

En nuestro Estudio se ha producido un Sesgo en el porcentaje de los Pacientes a los que no se les ha indicado Reincorporación Laboral, del 20\%. Porque a pesar de haber mejorado de forma significativa su Capacidad Funcional, en sus Antecedentes en relación a su Situación Laboral, ya estaban Prejubilados o eran subsidiarios de una Incapacidad Permanente previa.

Por lo que el porcentaje de Pacientes a los que se les podría haber indicado Reincorporación Laboral seria de un 83\%

Aplicando un Programa Educacional y Seguimiento desde Atención Primaria a los Pacientes de Bajo Riesgo, con precoz Reincorporación Laboral, o bien aplicando un Programa Completo de Rehabilitación a los Pacientes de Bajo-Medio Riesgo, Medio y Alto Riesgo, tras el cual y haber comprobado la mejora de todos los parámetros anteriormente establecidos se podría proceder a la indicación de Reincorporación Laboral.

La mayoría de los trabajos publicados sobre la Reincorporación Laboral de los Pacientes tras haber sufrido un SCA, coinciden en que la mayoría de los Pacientes de Bajo y Medio Riesgo podrían Reincorporarse a las 10 semanas del SCA (IAM).

Mientras que los de Alto Riesgo Cardiológico precisan una Evaluación más especifica, por si no fuera compatible con la Actividad Laboral del paciente.

Con todo esto, además de conseguir la mejoría Física, Psicológica del Paciente y Educacional (con Estilos de vida mas Cardiosaludables), también disminuiríamos los importantes Costes derivados de la Incapacidad Laboral (derivada de los largos periodos de Incapacidad Temporal o de la importante pérdida de productividad, a través de las Incapacidades Permanentes).

El estudio que hemos realizado muestra que La Rehabilitación Cardiaca, como una Intervención Multidisciplinar, que previene la progresión de la Aterosclerosis y de la Cardiopatía Isquémica en general, así como que mejora de forma significativa la Funcionalidad Cardiaca, con aumento de la Capacidad Funcional y de la Función Ventricular Izq. (FEVI), mejorando claramente también el Pronóstico y la Percepción por parte del Paciente de mejor Calidad de Vida; debería de ser una parte Integral del manejo Cardiológico después de sufrir un Evento Cardiaco Agudo o Crónico. 